\title{
Methods for Separation and Analysis of a Lactobacillus Biomass, Able to be Used for Health Improvement and Convalescence
}

\section{Ana Despina Ionescu $^{1 *}$, Nicoleta Rusu${ }^{1}$, Angela Casarica ${ }^{1}$, Roxana- Madalina Stoica ${ }^{1}$, Mariana- Gratiela Vladu ${ }^{1}$ and Emanuel Vamanu ${ }^{2}$ \\ ${ }^{1}$ National Institute for Chemical - Pharmaceutical Research and Development, Bucharest, Vitan Avenue, Romania \\ ${ }^{2}$ The University of Agricultural Sciences and Veterinary Medicine, Bucharest, Marasti} Boulevard, Romania

*Corresponding Author: Ana Despina Ionescu, National Institute for ChemicalPharmaceutical Research and Development, Bucharest, Vitan Avenue, Romania.
Received: February 24, 2020

Published: March 31, 2020

(C) All rights are reserved by Ana Despina Ionescu., et al.

\begin{abstract}
A set of chemical and microbiological analyses was established, in order to substantiate scientifically the valuable content of a Lactobacillus biomass, in order to use it as a component of a probiotic preparation playing an important role on health improvement and convalescence. The best results concerning the optical density of the cultures and the quality of the biological material were obtained using a culture of Lactobacillus paracasei on media with honey, pollen and raffinose.
\end{abstract}

Keywords: Lactobacillus; Biomass Analyses; Health Improvement

\section{Abbreviations \\ L. Paracasei: Lactobacillus paracasei; L. rhamnosus: Lactobacil- lus rhamnosus; L. Plantarum: Lactobacillus plantarum.}

\section{Introduction}

A set of biochemical and microbiological analyses was established, in order to substantiate scientifically the lactic-acid biosynthetic potential and the valuable content of a pure Lactobacillus biomass, in order to use it as a component of a probiotic preparation. This paper presents the selection of the methods most appropriate by which the characteristics of the bacterial biomass can be verified and used. Further the conditioned formula of the final probiotic product was represented by a cream and the best composition of this product was found by using some strains resistant to high sugar content, like a culture of Lactobacillus paracasei.

There are many other studies carried out all around the world, in order to obtain different probiotic preparations, depending on the local raw materials and the microbial strains adapted to the specific environmental conditions.

The conclusion of this study was that probiotic potential of the isolated probiotic L. rhamnosus and L. Plantarum is highest $(100 \%)$ as compared to commercially available probiotic preparations, such as Pre-Pro kid, Sporlac powder, LactoBacil plus, P-
Biotics kid and Gastroline containing probiotics. According to this research way, a better bacterial strain (L. Paracasei) was discover by our works.

\section{Materials and Methods}

The Lactobacillus probiotic strains selection was made in according with the following parameters: the strain development, the lactic-acid biosynthesis level and the utilization of sugar or its final concentration. In order to obtain a pure biomass preparation, 3 steps at centrifuge (each of them with increased speed) were used.

The next work of our studies was represented by the experiments concerning the highest rate of the cellular multiplication and the most valuable biomass composition (traced by the level of the present microelements) which may be obtained. The further operation was carried out in order to prepare the Lactobacillus probiotic biomass as necessary for the trace elements determination. A three- step scheme was selected for the biomass Microwave Digestion, depending on the organic compounds types and by using pure nitric acid as reagent. Ultrapure water with a specific resistance of $18 \mathrm{M} \Omega$ or greater was obtained using a Milli- $\mathrm{Q}^{\mathrm{TM}}$ water purification system (Millipore Corporation, USA).

Nitric acid (65\%, m/v, Ultrapure ${ }^{\circledR}$ grade, Merck, Darmstadt, Germany) was used. 
Standard solutions for calibration curves were prepared by dilutions of $10 \mathrm{mg} \mathrm{L}^{-1}$ of Standard 3 (trasable to NIST) procured from Perkin Elmer, Inc. Shelton, USA.

Microwave digestion of the Lactobacillus probiotic biomass samples for ICP-MS analysis was carried out using a model Multiwave $^{\text {TM }} 3000$ microwave system (Anton Paar).

The copper, zinc, cobalt, cadmium and iron total concentrations were determined by A Perkin Elmer Elan DRC-e inductively coupled plasma spectrometer ICP-MS and by FAAS [1-14].

\section{Results and Discussions}

The first studies, carried out on a laboratory scale, concerning the multiplication of some Lactobacillus and Bifidobacterium probiotic strains on media supplemented with honey and milled/ non-milled pollen, indicated the best results on media with honey supplemented also with other different oligosaccharides. Then, our studies were carried out in order to obtain a higher rate of the cellular multiplication and a more valuable biomass composition (traced by the level of the present microelements). Therefore, the copper, zinc, cobalt, cadmium and iron total concentrations were determined and the obtained results are presented in the next Table and Graphics and picture.

\begin{tabular}{|l|c|c|c|}
\hline Element & $\begin{array}{c}\text { Sample 1 } \\
\text { Lactobacillus } \\
\text { plantarum } \\
\text { (ppm) }\end{array}$ & $\begin{array}{c}\text { Sample 2 } \\
\text { Bifidobacterium } \\
\text { (ppm) }\end{array}$ & $\begin{array}{c}\text { Sample 3 } \\
\text { Lactobacillus } \\
\text { paracasei (ppm) }\end{array}$ \\
\hline $\mathrm{Cu}$ & 17,62 & 17,02 & 45,54 \\
\hline $\mathrm{Zn}$ & 38,59 & 37,98 & 62,18 \\
\hline $\mathrm{Co}$ & 5,05 & 5,02 & Under 1,0 \\
\hline $\mathrm{Cd}$ & 0,06 & 0,05 & 0,30 \\
\hline $\mathrm{Fe}$ & 668,96 & 664,24 & 183,1 \\
\hline
\end{tabular}

Table 1: The $\mathrm{Cu}, \mathrm{Zn}, \mathrm{Co}, \mathrm{Cd}$ and Fe Determination.

\section{Conclusions}

The data obtained by our researches indicate the best optical density (the parameter used for the strains development's determination) and the highest yields of lactic acid and some microelements, in the case of some bacterial strains initially selected on a fermentation media containing honey, human milk and raffinose.

The analyses of the biomass preparation obtained on a culture medium based on glucose, peptone and yeasts extract indicate a greater presence of the iron and zinc (important elements involved in health improvement and for accelerating the convalescence) mostly in the case of Lactobacillus paracasei selected strain.
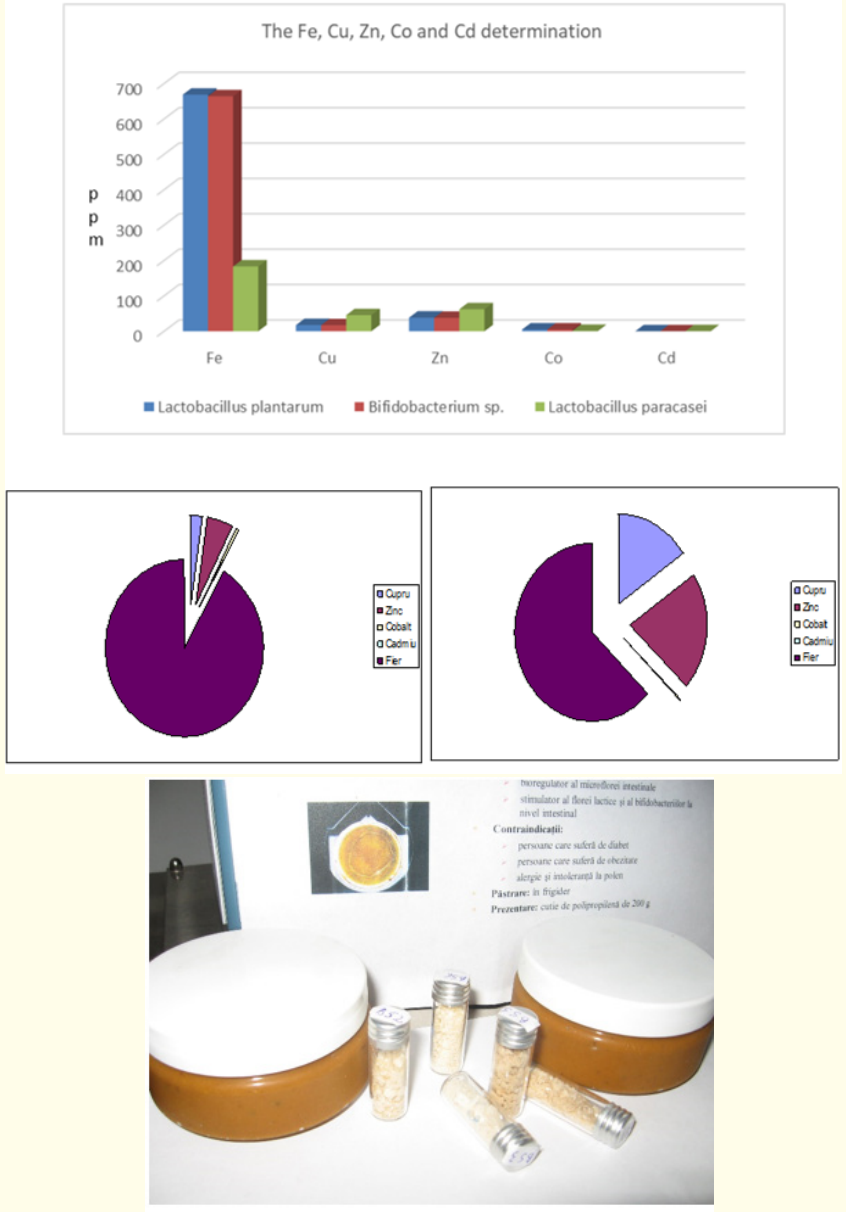

Figure: Biomass of Lactobacillus and the probiotic preparation.

The repeated analyses indicate a good stability of our pure biomass of Lactobacillus preparation.

\section{Bibliography}

1. Bogdański P and Schmidt M. "The Effect of Multispecies Probiotic Supplementation on Iron Status in Rats". Biological Trace Element Research 192.2 (2019).

2. Vitafoods Europe 2019 (Guest blogger). "Unlocking the full potential of probiotics" (2019).

3. IPA International probiotics association. "Iron Supplements Can Mess with Microbes" (2019).

4. Ying Yang and Zacchary Greenleaf. "Microaerobic fermentation of Lactobacillus acidophilus within Gut Microbiome Physiological conditions by BioFlo Bioprocess Control Stations". Eppendorf Application No. 412 (2019).

5. Yang Y and Sha M. "A beginner's guide to bioprocess mode: batch, fed-batch, and continuous fermentation". Eppendorf Application No. 408 (2019). 
6. Bussarin Kosin and Sudip Kumar Rakshit. "Microbial and Processing Criteria for Production of Probiotics". Food Technology and Biotechnology 44.3 (2006): 371379.

7. Oktay YERLIKAYA. "Starter cultures used in probiotic dairy product preparation and popular probiotic dairy drinks". Food Science and Technology Campinas 34.2 (2014): 221-229.

8. DH Tambekar and SA Bhutada. "An evaluation of probiotic potential of Lactobacillus sp. from milk of domestic animals and commercial available probiotic preparations in prevention of enteric bacterial infections". Recent Research in Science and Technology 2.10 (2010): 82-88.

9. Asma Manzoor and Javed Iqbal Qazi. "Significantly enhanced biomass production of a novel bio-therapeutic strain Lactobacillus plantarum (AS-14) by developing low cost media cultivation strategy". Journal of Biological Engineering 11 (2017).

10. EJ Agguire - Ezkauriatza and A Ramirez - Medrano. "Production of probiotic biomass (Lactobacillus casei) in goat milk whey: Comparison of batch, continuous and fed-batch cultures". Bioresource Technology 101.8 (2010): 2837-2844.

11. CP Chang and SL Llev. "Growth Medium Optimization for Biomass Production of a Probiotic Bacterium, Lactobacillus rhamnosus ATCC 7469". Journal of Food Biochemistry (2012).

12. Chin - Fa Hwang and Jen- Han Chang. "Optimization of medium composition for improving biomass production of Lactobacillus plantarum Pi06 using the Taguchi array design and the Box-Behnken method". Biotechnology and Bioprocess Engineering 17 (2012).

13. Chaline Caren Coghetto and Carolina Bettker Vasconcelos. "Lactobacillus plantarum BL011 cultivation in industrial isolated soybean protein acid residue". Brazilian Journal of Microbiology 47.7 (2016).

14. Lule WK and Tomar SK. "Bioavailability assessment of zinc enriched lactobacillus biomass in a human colon carcinoma cell line (Caco-2)". Food Chemistry 309 (2019).

\section{Assets from publication with us}

- Prompt Acknowledgement after receiving the article

- Thorough Double blinded peer review

- Rapid Publication

- Issue of Publication Certificate

- High visibility of your Published work

Website: https://www.actascientific.com/

Submit Article: https://www.actascientific.com/submission.php Email us: editor@actascientific.com

Contact us: +919182824667

Citation: Ana Despina Ionescu., et al. "Methods for Separation and Analysis of a Lactobacillus Biomass, Able to be Used for Health Improvement and Convalescence". Acta Scientific Microbiology 3.4 (2020): 185-187. 